\title{
Manifestações orais em pacientes com AIDS em uma população brasileira
}

\section{Oral manifestations in HIV - infected patients in a Brazilian population}

\author{
Lélia Batista de SOUZA* \\ Leão PEREIRA PINTO** \\ Ana Myrian Costa de MEDEIROS*** \\ Raimundo Fernandes de ARAÚJO Jr. **** \\ Olibânia Jácome Xavier de MESQUITA*****
}

\begin{abstract}
SOUZA, L. B.; PEREIRA PINTO, L.; MEDEIROS, A. M. C.; ARAUUO Jr., R. F.; MESQUITA, O. J. X. Manifestações orais em pacientes com AIDS em uma população brasileira. Pesq Odont Bras, v. 14, n. 1, p. 79-85, jan./mar. 2000.

Foram analisados 100 pacientes HIV+/AIDS no Hospital Giselda Trigueiro - Natal/RN, Brasil no período de 1996-97 quanto a presença de manifestações orais nestes pacientes. Setenta e quatro pacientes eram do sexo masculino e 26 do feminino. A idade média dos pacientes do sexo masculino foi 40 anos, variando de 24 a 67 anos e no feminino 29,8 anos variando de 17 a 48 anos. As manifestações orais mais freqüentes foram candidiase (homens - 79,7\%; mulheres $80,7 \%$ ), gengivite e periodontite (homens - 79,7\%; mulheres - 73,0\%), leucoplasia pilosa (homens - 6,7\%; mulheres 3,8\%), herpes labial (homens - 5,4\%; mulheres - 7,6\%), sarcoma de Kaposi (homens - 6,7\%).Verificou-se que 62,2\% dos homens eram homo- ou bissexuais e $100 \%$ das mulheres eram heterossexuais. Nossos resultados revelam semelhanças com outros trabalhos realizados em pacientes de outras regiões do mundo.
\end{abstract}

UNITERMOS: Sindrome da imunodeficiência adquirida; Manifestações bucais; Doenças da boca.

\section{INTRODUÇÃO}

A Sindrome da Imunodeficiência Adquirida (AIDS/SIDA) foi reconhecida oficialmente como doença em 1981, pelo Centros de Controle de Doenças e Prevenção (CDC) em função de uma explosão de casos inexplicados de sarcoma de Kaposi e de pneumonia por Pneumocystis carinni em homossexuais masculinos ocorridos em diversas cidades dos Estados Unidos.

Em 1986, a Comunidade Econômica Européia reunida em Copenhague discutiu os problemas orais relacionados com a infecção pelo HIV. Deste encontro resultou uma listagem de 30 doenças que apresentavam lesões associadas com a infecção pelo HIV. Posteriormente, foram acrescentadas novas lesões que ocorriam na boca ou região submandibular, a esta lista. Desta forma, as lesões foram classificadas onde incluíam-se lesões por infecção fúngicas, bacterianas e virais, além de processos neoplásicos e lesões de natureza desconhecida $^{2,16}$.

De acordo com AXELL; BAERT ${ }^{1}$ (1990), as manifestações bucais associadas com a infecção pelo HIV devem ser listadas dentro de três grupos, baseados na intensidade com que se apresentam nesta associação. O grupo I consiste de lesões orais que estão comumente associadas com infecção pelo HIV, como por exemplo candidíase, leucoplasia pilosa, gengivite úlcero-necrosante aguda (GUNA) e sarcoma de Kaposi. Ulcerações atípicas, doenças de glândulas salivares, infecções virais por citomegalovirus (CMV) e vírus herpes, papiloma vírus e varicela-zoster são enquadradas no grupo II, pois são menos comuns de ocorrerem. O grupo III abrange lesões possivelmente associadas com infecção pelo HIV, como exemplo osteomielite,

\footnotetext{
*Professor Doutor; ** Professor Doutor, Livre-Docente; **** Cirurgião-Dentista e Mestrando - Disciplina de Patologia Oral da Faculdade de Odontologia da Universidade Federal do Rio Grande do Norte.

*** Professora da Disciplina de Diagnóstico Oral e Doutoranda da Disciplina de Patologia Oral da Faculdade de Odontologia da Universidade Federal do Rio Grande do Norte

***** Bolsista de Iniciação Científica, PIBIC/CNPq/Universidade Federal do Rio Grande do Norte.
} 
SOUZA, L. B.; PEREIRA PINTO, L.; MEDEIROS, A. M. C.; ARAÚJO Jr., R. F.; MESQUITA, O. J. X. Manifestações orais em pacientes com AIDS em uma população brasileira. Pesq Odont Bras, v. 14, n. 1, p. 79-85, jan./mar. 2000.

distúrbios neurológicos, sinusite, carcinoma epidermóide, etc.

Diversos autores enfocaram em suas pesquisas, o estudo das manifestações orais de pacientes HIV+ / AIDS. Verifica-se na literatura que há predominância de alguns tipos de lesões onde a candidiase nas suas diversas apresentações clínicas, as doenças gengivais e/ou periodontais, a leucoplasia pilosa, o sarcoma de Kaposi e o herpes simples se situavam entre as mais freqüentemente citadas pelos autores ${ }^{1,2,3,5,6,10,11,12,15,16,17,18}$.

Achados de LIMA et al. ${ }^{11}$ (1994), verificaram que as infecções fúngicas se instalam em um grande número de pacientes HIV+, devido as profundas alterações que ocorrem na função imunológica mediada por linfócitos T. Dentre esses fungos patogênicos, a Candida albicans tem papel relevante no desencadeamento de infecções, apresentando-se como lesão inicial na freqüência de $3 \%$ a $30 \%$, sendo as formas mucosas as mais comuns e precoces.

CORREA; SUGAYA; BIRMAN ${ }^{4}$ (1994) consideram a leucoplasia pilosa um marcador clínico importante, cuja prevalência aumenta com a progressão da doença, chegando a $40 \%$ dos pacientes quando a contagem CD4 atinge $400 / \mathrm{mm}^{3}$.

GREESPAN; GREESPAN ${ }^{7}$ (1992) verificaram que esta foi a lesão mais prevalente em 198 pacientes africanos HIV+.

Dentre as lesões de origem bacteriana associadas à infecção pelo HIV estão a gengivite e periodontite de evolução rápida. Com a imunossupressão ocorrem alterações na microbiota normal da boca, notando-se um aumento das bactérias anaeróbias gram-negativas subgengivais, com conseqüente distúrbios nos componentes salivares, como por exemplo enzimas antimicrobianas e imunoglobulinas. Portanto, há uma diminuição da capacidade da saliva em controlar a microbiota e placa bacteriana propiciando o desenvolvimento de lesões gengivais e periodontais ${ }^{1,10}$.

De acordo com KENNEDY et al. ${ }^{8}$ (1999) o vírus herpes humano 8 (HHV-8) é atualmente reconhecido como um co-fator infeccioso na patogênese do sarcoma de Kaposi, embora não se saiba claramente o modo pelo qual o HHV-8 causa esta lesão. Provavelmente, ocorre conjunção com outros fatores incluindo-se citocinas, proteínas anti-apoptoses e receptores celulares de superficie.

Também OFFERMANN ${ }^{13}$ (1999) relata que o vírus herpes humano tipo 8 (HHV-8) está implicado como agente etiológico do sarcoma de Kaposi; se- gundo ainda este autor, tantos os monócitos como os linfócitos B aparecem como reservatórios para o HHV-8 em indivíduos assintomáticos, considerando que o HHV-8 está presente nas células escamosas e células endoteliais microvasculares dentro das lesões de sarcoma de Kaposi.

Em diversas publicações sobre o tema verifica-se que, quando são analisadas as variáveis sexo e idade destes pacientes infectados pelo HIV, embora possa ocorrer em qualquer faixa etária e em ambos os sexos, há uma maior freqüência em pacientes do sexo masculino ${ }^{1,6,10,15,16}$ e pacientes jovens, predominantemente nas $3^{a}$ e $4^{a}$ faixas de idade $^{2,6,10,12,15}$.

No estudo desses pacientes, grande número de autores procuram analisar o comportamento de risco dos mesmos, uma vez que este é um fator de fundamental importância para o desenvolvimento da doença e, dentro deste contexto, são divididos quanto a esta variável nos grupos sanguíneo, sexual e usuários de drogas endovenosas.

Em alguns estudos têm-se verificado que os pacientes categorizados como usuários de drogas endovenosas, representam os mais freqüentemente infectados pelo HIV [BARONE et al. ${ }^{2}$ (1990), CEBALLOS-SALOBREÑA; AGUIRRE-URIZAR, BAGAN-SEBATIAN $^{3}$ (1996) e MONIACI et al. ${ }^{12}$ (1990)], embora outros trabalhos indiquem como sendo os mais acometidos pela doença, homens do grupo homo-/ bissexuais ${ }^{10,15,17}$.

LAMSTER et al. ${ }^{9}$ (1994) revelaram que as manifestações bucais mais encontradas em homossexuais norte-americanos soropositivos não foram as mesmas observadas nos usuários de droga endovenosas soropositivos. Dentre outras, foram avaliadas variáveis como idade, raça e experiência com fumo. Estes pesquisadores propuseram, ainda, que entre outros parâmetros, deve-se observar: hábitos e costumes do grupo estudado; condição sócio-econômica; nivel cultural; condições geográficas da região estudada; época de exposição ao vírus; forma de contágio do grupo estudado; condições de higiene bucal; medicamentos utilizados; pesquisa de doença sistêmica prévia e estilo de vida. Portanto, para estes autores, estas variáveis podem influenciar no desenvolvimento de certas lesões bucais, em pessoas com infecção pelo HIV, em determinadas regiões geográficas.

\section{MATERIAL E MÉTODOS}

No presente estudo, foram examinados $100 \mathrm{pa}-$ cientes internados no setor de AIDS do Hospital 
SOUZA, L. B.; PEREIRA PINTO, L.; MEDEIROS, A. M. C.; ARAÚJO Jr., R. F.; MESQUITA, O. J. X. Manifestações orais em pacientes com AIDS em uma população brasileira. Pesq Odont Bras, v. 14, n. 1, p. 79-85, jan./mar. 2000.

Giselda Trigueiro em Natal/RN, Brasil, todos portadores de AIDS. Além do exame clínico geral e exame da cavidade oral, foram também aplicados questionários específicos, objetivando colher informações do paciente quanto ao seu comportamento de risco.

Foram obtidas informações quanto as variáveis sexo, idade desses pacientes, além da avaliação das estruturas orais e periorais para detecção das manifestações presentes nesses pacientes, bem como a presença ou não de doença sistêmica e período transcorrido entre o diagnóstico sorológico e o aparecimento das manifestações clínicas.

Os dados foram obtidos tanto durante o exame clínico como também através dos prontuários desses pacientes.

\section{RESULTADOS}

Dos 100 pacientes estudados, 74 eram do sexo masculino e 26 do feminino. Com relação a idade desses pacientes, verificou-se que a média para aqueles do sexo masculino foi 40 anos, com idade variando de 24 a 67 anos e, no sexo feminino, 29,8 anos, variando de 17 a 48 anos.

Os dados referentes ao comportamento de risco encontram-se presentes na Tabela 1.

Com relação a presença de manifestações orais nesses pacientes, verificou-se a ocorrência de candidiase, nas suas diversas apresentações clínicas, leucoplasia pilosa, sarcoma de Kaposi, herpes simples, GUNA, gengivite, periodontite e úlcera tuberculosa (Tabela 2).

Quando analisamos a distribuição das lesões de candidiase nas diversas apresentações clínicas, verificamos que o tipo pseudomembranoso esteve presente em 34 pacientes do sexo masculino e 10 no feminino, seguido da queilite angular com 23

TABELA 1 - Distribuição em números absolutos dos casos de AIDS, segundo o sexo dos pacientes e categoria de risco. Natal/RN, 1996-1997.

\begin{tabular}{c|c|c|c|c|c}
\hline \hline \multirow{2}{*}{ Sexo } & \multicolumn{3}{c}{ Cexual } & \multicolumn{2}{c}{ Sanguíneo } \\
\cline { 2 - 6 } & $\begin{array}{c}\text { Homos- } \\
\text { sexual }\end{array}$ & Bissexual & $\begin{array}{c}\text { Heteros- } \\
\text { sexual }\end{array}$ & UDE* & $\begin{array}{c}\text { Trans- } \\
\text { fundido }\end{array}$ \\
\hline Masculino & 19 & 27 & 23 & 6 & 5 \\
\hline Feminino & - & - & 26 & - & - \\
\hline Total & 19 & 27 & 49 & 6 & 5 \\
\hline \hline
\end{tabular}

Fonte: Hospital Giselda Trigueiro - Natal/RN.

*Usuários de Drogas Endovenosas. casos em pacientes do sexo masculino e 7 no feminino. O tipo eritematoso foi constatado em 11 casos de pacientes do sexo masculino e 5, no feminino. Ocorreram 4 casos do tipo hiperplásico, todos em pacientes do sexo masculino.

Os dados referentes à distribuição das manifestações orais, quanto ao sexo dos pacientes, encontram-se expressos na Tabela 2.

Os dados referentes à correlação entre as variáveis manifestações orais e comportamentos de risco encontram-se na Tabela 3.

A presença de algumas manifestações sistêmicas nesses pacientes foi constatada e, dentre estas, destacam-se a diarréia crônica, pneumonia, tuberculose pulmonar, toxoplasmose, astenia e mialgia, úlcera gástrica, tuberculose ganglionar, sífilis e cefaléia frontal.

No presente trabalho, procuramos também analisar o tempo transcorrido para o desenvolvimento da doença AIDS após o diagnóstico da infecção através dos exames sorológicos e sua correlação com os comportamentos de risco desses pacientes. Foi verificado que no grupo masculino, os homossexuais e bissexuais necessitaram de 2,5 anos para o desenvolvimento da doença; os heterossexuais, em 2,3 anos.

Quando se correlaciona esta variável com fatores sanguíneos e usuários de drogas endovenosas verificou-se que estes últimos pacientes necessitaram de 2,1 anos, enquanto os transfundidos, 3,2 anos.

TABELA 2 - Distribuição em números absolutos das manifestações orais de pacientes com AIDS, segundo a variável sexo. Natal/RN, 1996-1997.

\begin{tabular}{l|c|c}
\hline \hline \multirow{2}{*}{ Manifestações orais } & \multicolumn{2}{|c}{ Sexo } \\
\cline { 2 - 3 } & Masculino & Feminino \\
\hline Candidíase & 72 & 21 \\
\hline Leucoplasia pilosa & 5 & 1 \\
\hline Sarcoma de Kaposi & 5 & - \\
\hline Herpes Simples & 4 & 2 \\
\hline GUNA & 4 & - \\
\hline Gengivite & 30 & 11 \\
\hline Periodontite & 29 & 8 \\
\hline Úlcera Tuberculóide & 1 & - \\
\hline Nenhuma & 4 & 4 \\
\hline Total & 154 & 47 \\
\hline \hline
\end{tabular}

Fonte: Hospital Giselda Trigueiro - Natal/RN. 
SOUZA, L. B.; PEREIRA PINTO, L.; MEDEIROS, A. M. C.; ARAÚJO Jr., R. F.; MESQUITA, O. J. X. Manifestações orais em pacientes com AIDS em uma população brasileira. Pesq Odont Bras, v. 14, n. 1, p. 79-85, jan./mar. 2000.

TABELA 3 - Distribuição em números absolutos das manifestações orais da AIDS em pacientes do sexo masculino, segundo o comportamento de risco. Natal/RN, 1996-1997.

\begin{tabular}{|c|c|c|c|c|c|c|}
\hline \multirow{3}{*}{$\begin{array}{l}\text { Manifesta- } \\
\text { ções Orais }\end{array}$} & \multicolumn{5}{|c|}{ Comportamento de risco } & \multirow{3}{*}{ Total } \\
\hline & \multicolumn{3}{|c|}{ Sexual } & \multicolumn{2}{|c|}{ Sanguíneo } & \\
\hline & $\begin{array}{c}\text { Homos- } \\
\text { sexual }\end{array}$ & $\begin{array}{c}\text { Bisse- } \\
\text { xual }\end{array}$ & $\begin{array}{c}\text { Heteros- } \\
\text { sexual }\end{array}$ & UDE* & $\begin{array}{l}\text { Trans- } \\
\text { fundido }\end{array}$ & \\
\hline Candidíase & 20 & 29 & 21 & 5 & 3 & 78 \\
\hline $\begin{array}{l}\text { Leucopla- } \\
\text { sia pilosa }\end{array}$ & 1 & 3 & 1 & 2 & - & 7 \\
\hline $\begin{array}{l}\text { Sarcoma } \\
\text { de Kaposi }\end{array}$ & 4 & 1 & - & - & 1 & 6 \\
\hline $\begin{array}{l}\text { Herpes } \\
\text { simples }\end{array}$ & 1 & 1 & 2 & 1 & - & 5 \\
\hline GUNA & 1 & 2 & 1 & - & - & 4 \\
\hline Gengivite & 8 & 12 & 4 & 3 & 1 & 28 \\
\hline $\begin{array}{l}\text { Periodon- } \\
\text { tite }\end{array}$ & 5 & 8 & 13 & 2 & 3 & 31 \\
\hline $\begin{array}{l}\text { Úlcera tu- } \\
\text { berculóide }\end{array}$ & 1 & - & - & - & - & 1 \\
\hline Nenhuma & 1 & - & - & 1 & 1 & 3 \\
\hline Total & 42 & 56 & 42 & 14 & 9 & 163 \\
\hline
\end{tabular}

Fonte: Hospital Giselda Trigueiro - Natal/RN.

*Usuários de Drogas Endovenosas.

Ao analisarmos o tempo transcorrido para o desenvolvimento da AIDS nos pacientes do sexo feminino foi verificado que este correspondeu a 1,7 anos.

$\mathrm{Na}$ distribuição dos achados quanto a localização anatômica destas lesões foi constatado que, nos pacientes do sexo masculino, as lesões por candidiase estiveram presentes no dorso e borda lateral da língua, mucosa jugal e labial e palato, tanto no palato duro como no palato mole; os casos de sarcoma de Kaposi ocorreram no palato mole (02 casos) e, duro (01 caso), gengiva (01 caso) e pele (01 caso). Os lábios foram sede das lesões de herpes simples e úlcera tuberculosa. Os casos de leucoplasia pilosa ocorreram na mucosa da borda lateral da língua, bilateralmente.

Nas pacientes dos sexo feminino, a candidiase ocorreu no dorso e borda lateral da língua, mucosa jugal, mucosa labial e palato duro e mole; não houve nenhum caso de sarcoma de Kaposi, como também úlcera tuberculosa. Os casos de herpes foram
TABELA 4 - Distribuição em números absolutos das manifestações orais da AIDS em pacientes do sexo feminino, segundo o comportamento de risco. Natal/RN, 1996-1997.

\begin{tabular}{l|c|c|c}
\hline \multirow{2}{*}{$\begin{array}{c}\text { Manifestações } \\
\text { Orais }\end{array}$} & \multicolumn{3}{|c}{ Comportamento de risco } \\
\cline { 2 - 4 } & $\begin{array}{c}\text { Homoss. e } \\
\text { bissexual }\end{array}$ & $\begin{array}{c}\text { Heteros- } \\
\text { sexual }\end{array}$ & $\begin{array}{c}\text { Hemof./ } \\
\text { transf./UDE* }\end{array}$ \\
\cline { 2 - 4 } Candidose oral & - & 21 & - \\
\hline Leucoplasia pilosa & - & 1 & - \\
\hline Sarcoma de Kaposi & - & - & - \\
\hline Herpes simples & - & 2 & - \\
\hline Guna & - & - & - \\
\hline Gengivite & - & 11 & - \\
\hline Periodontite & - & 8 & - \\
\hline Úlcera tuberculóide & - & - & - \\
\hline N.D.N. & - & 4 & - \\
\hline Total & - & 47 & Sanguíneo \\
\hline \hline
\end{tabular}

Fonte: Hospital Giselda Trigueiro - Natal/RN.

*Usuários de Drogas Endovenosas.

labiais, e um caso de leucoplasia pilosa, ocorreu na da borda lateral da lingua, bilateralmente.

Neste grupo foi evidenciado a presença de manifestações sistêmicas como: diarréia, pneumonia, tuberculose pulmonar, toxoplasmose, astenia, anorexia, tuberculose ganglionar, úlcera gástrica, cefaléia frontal e dispnéia.

\section{DISCUSSÃO}

A Sindrome da Imunodeficiência Adquirida tem merecido maior atenção por parte dos pesquisadores e dos órgãos de saúde, de um modo geral, em virtude de sua gravidade. Isto decorre não só pelo indice de mortalidade que ela provoca mas, também, pelos diversos aspectos sociais, econômicos e de saúde pública que estão a ela associados.

A presença de lesões orais em pacientes com AIDS tem sido motivo de diversos trabalhos ${ }^{1,2,11,16,18}$, tendo em vista que, essas manifestações orais representam os primeiros sinais da doença. Por este motivo é de fundamental importância o conhecimento detalhado das lesões orais presentes nesses pacientes, como forma do cirurgião-dentista identificar, o mais precocemente possivel, os casos de pacientes com AIDS nas suas atividades clínicas. 
SOUZA, L. B.; PEREIRA PINTO, L.; MEDEIROS, A. M. C.; ARAÚJO Jr., R. F.; MESQUITA, O. J. X. Manifestações orais em pacientes com AIDS em uma população brasileira. Pesq Odont Bras, v. 14, n. 1, p. 79-85, jan./mar. 2000.

Com o reconhecimento oficial desta sindrome, como doença, e a constatação de manifestações orais que devem ser reconhecidas pelos profissionais que atuam na área, foi que motivou a realização de vários trabalhos, no sentido de estabelecer critérios para o reconhecimento de lesões associadas e sua freqüência ${ }^{2,16,18}$.

Ficou demonstrado que, de modo geral, as lesões orais presentes nesses pacientes correspondem àquelas por infecções fúngicas, bacterianas e virais, além de processos neoplásicos e outras entidades de etiologia desconhecida.

A freqüência com que essas lesões se manifestam nos pacientes, sofre algumas variações, muitas delas em função de hábitos individuais, fatores sociais ou mesmo, geográficos ${ }^{9,17}$.

Outro aspecto bastante relatado na literatura diz respeito a correlação que tem sido estabelecida entre o tipo de lesão presente e o comportamento de risco dos pacientes: sexual, sanguíneo e usuário de drogas endovenosas. No grupo sexual se enquadram os pacientes homossexuais, bissexuais ou heterossexuais e no grupo sanguíneo estão os hemofilicos e transfundidos sanguíneos.

Dentre as lesões presentes nesses pacientes, destaca-se a candidiase nas suas diversas formas clínicas, seguido por leucoplasia pilosa, gengivites e/ou periodontites progressivas, herpes simples, sarcoma de Kaposi, lesões do papiloma virus humano (condiloma acuminado, verruga vulgar e hiperplasia epitelial focal), entre outras $^{1,2,5,6,10,11,12,14,15,16,17,18}$.

Em nosso estudo verificamos que dos 100 pacientes analisados, naquele período de tempo, houve predominância de pacientes do sexo masculino (74 casos) sobre o feminino (26 casos), achados estes semelhantes aos de outros autores ${ }^{2,3,6,10,12,14,15,17}$.

A candidiase oral foi a manifestação mais freqüente com 99 ocorrências sendo que, desse total, aconteceram $78 \mathrm{em}$ pacientes do sexo masculino e, 21, no feminino. Destacamos que esta entidade se apresentou nas diversas formas clinicas: pseudomembranosa, eritematosa, hiperplásica e do tipo queilite angular. A pseudomembranosa foi a forma mais freqüente, semelhante aos achados de LASKARIS, HADJIVASSILIOU, STRATIGOS ${ }^{10}$ (1992). Outros achados clínicos bastante freqüentes em nosso estudo, foram as lesões de gengivite e periodontite com 39 ocorrências de cada modalidade. Estes achados são bastante semelhantes àqueles descritos na literatura ${ }^{1,3,10,16,17,18}$. Alguns au- tores encontraram, inclusive, uma maior freqüência de lesões periodontais do que os outros tipos de lesões ${ }^{3}$.

Verificamos que nos pacientes do sexo masculino, a idade média foi de 40 anos, variando dos 24 aos 67 anos; para os pacientes do sexo feminino a idade média foi 29,8 anos, com idade que variou dos 17 aos 48 anos. Nos pacientes do sexo feminino, a AIDS, acomete pessoas mais jovens, acarretando uma idade média mais baixa quando comparamos aos casos ocorridos nos do sexo masculino.

Um fato que chama a atenção é a correlação que se observa entre comportamento de risco e a ocorrência de determinados tipos de lesões orais. Em nossa amostra, os grupos de pacientes homossexuais e bissexuais foram os que apresentaram maior número de manifestações orais, particularmente a candidiase enquanto, nos usuários de drogas ou receptores de sangue, esse achado foi menos freqüente. Estes achados são semelhantes a estudos realizados em populações de outros países americanos ${ }^{6,9,15}$, porém diferentes daqueles realizados na Europa, onde as manifestações orais são mais freqüentes em grupos usuários de drogas endovenosas $^{2,3,12}$. No entanto, pelos estudos de SCHULTEN; tem KATE; van der WAARL ${ }^{17}$ (1989), LASKARIS; HADJIVASSILIOU; STRATIGOS ${ }^{10}$ (1992) e PORTER et al. ${ }^{14}$ (1989), trabalhando com amostragem de pacientes europeus, demonstraram resultados diferentes, uma vez que em seus achados, a maioria dos pacientes pertencia a categoria de risco homo-/bissexuais.

Nas pacientes do sexo feminino verificamos algumas diferenças dos dados encontrados em relação aos do sexo masculino, haja vista que naqueles a ocorrência da AIDS se relaciona, muito mais freqüentemente, com contaminação através do contato sexual.

Em nosso estudo procuramos analisar o tempo transcorrido entre a confirmação diagnóstica da infecção pelo HIV e a presença de manifestação clínica da doença AIDS, ou seja, o período para o desenvolvimento clínico da doença. Verificamos que nos pacientes do sexo masculino o tempo médio foi de 21 meses. Correlacionando este dado com o comportamento de risco, verificamos que nos usuários de drogas endovenosas este tempo foi menor (21 meses), enquanto nos transfundidos foi o maior intervalo (32 meses). Nas pacientes do sexo feminino, verificou-se que o tempo médio 
SOUZA, L. B.; PEREIRA PINTO, L.; MEDEIROS, A. M. C.; ARAÚJO Jr., R. F.; MESQUITA, O. J. X. Manifestações orais em pacientes com AIDS em uma população brasileira. Pesq Odont Bras, v. 14, n. 1, p. 79-85, jan./mar. 2000.

para desenvolver a doença foi 17 meses, configurando o fato de que as mulheres além de serem acometidas mais cedo pela AIDS, também, necessitam de um menor intervalo de tempo para o seu desenvolvimento com manifestações clínicas.

Do presente estudo depreende-se que as manifestações orais em pacientes com AIDS representam uma parcela importante dos sinais e sintomas da doença. Esta afirmação ratifica o que já foi observado em trabalhos anteriores, realizados por diversos pesquisadores em diferentes populações. Este fato pode estar associado a fatores diversos, que interferem na prevalência de determinados tipos de lesões. Essas variações, portanto, podem estar associados a alguns fatores como distribuição geográfica dos pacientes analisados, compor- tamento de risco desses pacientes, condições sócio-econômicas, entre outros.

\section{CONCLUSÕES}

1. A cavidade oral representa área de freqüentes manifestações da AIDS, sendo que a candidiase e as doenças periodontais representaram as lesões mais ocorrentes.

2. Os pacientes do sexo masculino foram mais acometidos pela doença, embora os do sexo feminino, quando afetados tenham mostrado o envolvimento em idade mais precoce.

3. Considerando a variável comportamento de risco, verificamos que houve uma maior freqüência em pacientes masculinos homo- ou bissexuais.

SOUZA, L. B.; PEREIRA PINTO, L.; MEDEIROS, A. M. C.; ARAÚJO Jr., R. F.; MESQUITA, O. J. X. Oral manifestations in HIV-infected patients in a Brazilian population. Pesq Odont Bras, v. 14, n. 1, p. 79-85, jan./mar. 2000.

One hundred $\mathrm{HIV}^{+}$/AIDS Brazilian patients (74 men and 26 women) were examined in the Giselda Trigueiro Hospital, in Natal, Rio Grande do Norte, Brazil in the period from 1996 to 1997 . The men's mean age was 40 years, ranging from 24 to 67 years and the women's was 29.8 years, ranging from 17 to 48 years. One or more oral findings were observed in theses patients. The most common oral lesions were candidiasis (men - 97.2\%; women - 80.7\%), gingivitis and periodontitis (men - 79.7\%; women - 7.3\%), hairy leukoplakia (men - 6.7\%; women - 3.8\%), herpes simplex (men $5.4 \%$; women - 2.7\%), and Kaposi's sarcoma (men - 6.7\%). From the men, $62.1 \%$ were homo-/bisexual, and $100 \%$ of the women were heterosexual.

UNITERMS: Acquired immunodeficiency syndrome; Oral manifestations; Mouth diseases.

\section{REFERÊNCIAS BIBLIOGRÁFICAS}

1. AXELL, T.; BAERT, S.; BROCHERIOU, C.; CHALLA COMBE, S.; GREENSPAN, D.; ten KATE, R. W.; LASKARIS, G.; MANO AZUL, A. PINDBORG, J. J.; REICHART, P. A.; SCHULTEN, E. A. J. M.; SCULLY, C.; SYRJANEN, S.; WILLIAMS, D.; van der WAAL, I. O. Revised classification of HIV - associated oral lesions. Brit den J, v. 170, n. 8, p. 305-6, April 1991.

2. BARONE, R.; FICARRA, G.; GAGLIOTI, D.; ORSI, A.; MAZZOTTA, F. Prevalence of oral lesions among HIV infected intravenous drug abusers and other risk groups. Oral Surg, v. 69, n.2, p. 169-73, Feb. 1990.

3. CEBALLOS-SALOBREÑA, A.; AGUIRRE-URIZAR, J. M.; BAGAN-SEBASTIAN, J. V. Oral manifestations associated with human immunodeficiency virus infection in a Spanish population. J Oral Pathol Med, v. 25, p. 523-6, 1996.

4. CORREA, O. C. L.; SUGAYA, N. N.; BIRMAN, E. G. Manifestações bucais de origem infecciosa em pacientes HIV positivos ou com AIDS. Parte II. Doenças virais (viróticas). Rev ABO Nac, v. 2, n.2, p. 105-8, abril/maio 1994.

5. EPSTEIN, J.; SCULLY, C. HIV infection: clinical features and treatment of thirty-three homossexual men with
Kaposi's sarcoma. Oral Surg, v. 71, n. 1, p. 38-41, Jan. 1991.

6. FEIGAL, D. W.; KATZ, M. H.; GREENSPAN, D.; WESTENHOUSE, J.; WINKELSTEIN Jr., W.; LANG, W.; SAMUEL, M.; BUCHBINDER, S. P.; HESSOL, N. A.; LIFSON, A. R.; RUTHERFORD, G. W.; MOSS, A.; OSMOND, D.; SHIBOSKI, S.; GREENSPAN, J. S. The prevalence of oral lesions in HIV - infected homossexual and bissexual men: three San Francisco epidemiological cohorts. AIDS, v. 5, n. 5, p. 519-25, 1991.

7. GREENSPAN, D.; GREENSPAN, J. S. Significance of oral hairy leukoplakia. Oral Surg, v. 73, p. 151-4, Feb. 1992.

8. KENNEDY, M. M., BIDDOLPH, S.; LUCAS, S. B.; HOWELLS, D. D.; PICTON, S.; McGEE, J. O.; O'LEARY, J. J. CD40 upregulation is independent of HHV-8 in the pathogenis of Kaposi's Sarcoma. Mol Pathol, v. 52, n. 1, p. 32-6, Feb. 1999.

9. LAMSTER, I. B.; BEGG, M. D.; MITCHELL-LEWIS, D.; FINE, J. B.; GRBIC, J. T.; TODAK, G. G.; EL-SADR, W.; GORMAN, J. M.; ZAMBON, J. J.; PHELAN, J. A. Oral manifestations of HIV infection in homossexual men and intravenous drug users. Oral Surg, v. 78, n.2, p. 163-223, Aug. 1994.

10. LASKARIS, G.; HADJIVASSILIOU, M.; STRATIGOS, J. Oral 
SOUZA, L. B.; PEREIRA PINTO, L.; MEDEIROS, A. M. C.; ARAÚJO Jr., R. F.; MESQUITA, O. J. X. Manifestações orais em pacientes com AIDS em uma população brasileira. Pesq Odont Bras, v. 14, n. 1, p. 79-85, jan./mar. 2000.

signs and symptoms in $160 \mathrm{HIV}$ - infected patients. J Oral Pathol Med, v. 21, p. 120-3, 1992.

11. LIMA, O. C. C.; SILVEIRA, F. R. X. da; BIRMAN, E. G. Manifestações bucais de origem infecciosa em pacientes HIV-positivos ou com AIDS I: doenças fúngicas. Rev ABO Nac, v. 2, n. 1, p. 28-32, fev/mar. 1994.

12. MONIACI, D.; GRECO, D.; FLECCHIA, G.; RAITERI, R. O.; SINICCO, A. Epidemiology, clinical features and prognostic value of HIV-1 related oral lesions. J Oral Pathol Med, v. 19, p. 477-81, 1990.

13. OFFERMANN, M. K. Consideration of host-viral interaction in the pathogenis of Kaposi's Sarcoma. J Acquir Immune Defic Syndr, v. 21, suppl 1, p. 558-65, August Review, 1999.

14. PORTER, S. R.; LUKER, J.; SCULLY, C.; GLOVER, S.; GRIFFITHS, M. J. Orofacial manifestations of a group of
British patients infected with HIV-1. J Oral Pathol Med., v. 18, n. 1, p. 47-8. 1989.

15. RAMIREZ, V.; GONZALEZ, A.; de la ROSA, E.; GONZALEZ, M.; RIVIERA, I.; HERNANDEZ, C.; PONCE DE LEON, S. Oral lesions in Mexican HIV - infected patients. J Oral Pathol Med., v. 19, p. 482-5, 1990.

16. SCHIØDT, M.; PINDBORG, J. J. AIDS and the oral cavity epidemiology and clinical oral manifestations of human immudeficiency virus infection: a review. Int J Oral Maxillofac Surg, v. 16, p. 1-14, 1987.

17. SCHULTEN, E. A. J. M.; tem KATE, R. W.; van der WAAL, I. Oral manifestations of HIV infection in 75 Dutch patients. J Oral Pathol Med, v. 18, n. 1, p. 42-6, Jan. 1989.

18. WILLIAMS, C. A.; WINKLER, J. R.; GRASSI, M.; MURRAY, P. A. HIV - associated periodontitis complicated by necrotizing stomatitis. Oral Surg, v. 69 , n. 3, p. 351-5, Mar. 1990.

Recebido para publicação em 20/06/99 Enviado para reformulação em 15/08/99 Aceito para publicação em 09/01/00 